\title{
Phytoprotection
}

\section{Évaluation de lignées de sorgho (Sorghum bicolor) pour leur résistance à la mauvaise herbe parasite Striga hermonthica au Burkina Faso}

\author{
A. Oliver, K.V. Ramaiah et G.D. Leroux
}

Volume 73, numéro 1, 1992

URI : https://id.erudit.org/iderudit/706016ar

DOI : https://doi.org/10.7202/706016ar

Aller au sommaire du numéro

Éditeur(s)

Société de protection des plantes du Québec (SPPQ)l

ISSN

0031-9511 (imprimé)

1710-1603 (numérique)

Découvrir la revue

Citer cet article

Oliver, A., Ramaiah, K. \& Leroux, G. (1992). Évaluation de lignées de sorgho (Sorghum bicolor) pour leur résistance à la mauvaise herbe parasite Striga hermonthica au Burkina Faso. Phytoprotection, 73(1), 13-23.

https://doi.org/10.7202/706016ar
Résumé de l'article

Des essais en pots et au champ ont été réalisés au Burkina Faso en 1987 pour évaluer la résistance de lignées de sorgho (Sorghum bicolor) vis-à-vis la mauvaise herbe parasite Striga hermonthica. Dans l'expérience en pots, les lignées de cycle court ICSV-1079 BF et ICSV-1078 BF ont présenté un nombre et une biomasse de $S$. hermonthica inférieurs à ceux du témoin sensible. Cette réaction n'a pas été confirmée dans l'expérience au champ. Aucune lignée ne s'est avérée résistante. Parmi les lignées de cycle moyen, aucune lignée ne s'est démarquée du témoin sensible dans l'expérience en pots. Dans l'expérience au champ, la lignée ICSV-1089 BF a présenté une faible sensibilité au $S$.

hermonthica. Cependant, cette lignée a obtenu de faibles rendements. Aucune des lignées testées ne semble pouvoir être recommandée pour la lutte envers le S. hermonthica dans le sorgho au Sahel. Le cultivar Framida ne saurait être utilisé comme source de résistance au $S$. hermonthica. Il est urgent d'identifier de nouvelles sources de résistance afin de répondre aux besoins des programmes d'amélioration du sorgho. 


\title{
Évaluation de lignées de sorgho (Sorghum bicolor) pour leur résistance à la mauvaise herbe parasite Striga her- monthica au Burkina Faso
}

\author{
Alain Olivier ${ }^{1}$, K.V. Ramaiah² et Gilles D. Leroux ${ }^{1}$
}

Reçu 1991-06-04; accepté 1992-01-24

Des essais en pots et au champ ont été réalisés au Burkina Faso en 1987 pour évaluer la résistance de lignées de sorgho (Sorghum bicolor) vis-à-vis la mauvaise herbe parasite Striga hermonthica. Dans I'expérience en pots, les lignées de cycle court ICSV-1079 BF et ICSV-1078 BF ont présenté un nombre et une biomasse de $S$. hermonthica inférieurs à ceux du témoin sensible. Cette réaction n'a pas été confirmée dans l'expérience au champ. Aucune lignée ne s'est avérée résistante. Parmi les lignées de cycle moyen, aucune lignée ne s'est démarquée du témoin sensible dans l'expérience en pots. Dans l'expérience au champ, la lignée ICSV-1089 BF a présenté une faible sensibilité au S. hermonthica. Cependant, cette lignée a obtenu de faibles rendements. Aucune des lignées testées ne semble pouvoir être recommandée pour la lutte envers le $S$. hermonthica dans le sorgho au Sahel. Le cultivar Framida ne saurait être utilisé comme source de résistance au $S$. hermonthica. II est urgent d'identifier de nouvelles sources de résistance afin de répondre aux besoins des programmes d'amélioration du sorgho.

Olivier, A., K.V. Ramaiah, and G.D. Leroux. 1992. Assessment of the resistance of sorghum (Sorghum bicolor) lines to the parasitic weed Striga hermonthica in Burkina Faso. PHYTOPROTECTION 73: 13-23.

Pot and field experiments were performed in Burkina Faso in 1987 in order to evaluate sorghum (Sorghum bicolor) lines for their resistance to the parasitic weed Striga hermonthica. In pot experiments, low duration lines ICSV-1079 BF and ICSV-1078 BF presented a lower number and biomass of S. hermonthica than the susceptible check. This reaction was not confirmed in field experiments. No line was resistant. Among medium duration lines, no line was significantly different from the susceptible check in pot experiments. ICSV-1089 BF exhibited a low susceptibility to $S$. hermonthica attack in field experiments. However, its yield was low. No line could be recommended in the perspective of controlling $S$. hermonthica in sorghum in Sahelian countries. The cultivar Framida is not an appropriate source of resistance to $S$. hermonthica. Other sources of resistance are urgently required to satisfy the needs of sorghum breeding programs.

\section{INTRODUCTION}

1. Département de phytologie, Faculté des sciences de l'agriculture et de l'alimentation, Université Laval, Québec (Québec), Canada G1K 7P4

2. International Crops Research Institute for the Semi-Arid Tropics, B.P. 320, Bamako, Mali
Le sorgho [Sorghum bicolor (L.) Moench] est une des principales cultures céréalières d'Afrique. II constitue la base de l'alimentation quotidienne de plusieurs millions de paysans des zones tropicales semi-arides. Son rendement moyen en 
Afrique demeure cependant très faible. Un des principaux facteurs responsables de ces faibles rendements est la présence de la mauvaise herbe parasite Striga hermonthica (Del.) Benth., qui occasionne chaque année des dommages évalués à 2,9 milliards de dollars U.S. (Saueborn 1991). Une infestation de Striga peut être catastrophique pour le paysan, entraînant des pertes de rendement atteignant parfois 70\% (Doggett 1988).

Plusieurs méthodes ont été envisagées pour lutter contre le parasite. Parmi celles-ci, l'utilisation de cultivars résistants suscite bien des espoirs. L'existence de différences variétales quant à la sensibilité du sorgho au $S$. hermonthica a été mise en évidence il y a plusieurs années (Doggett 1952, 1965; Wilson-Jones 1953). Cependant, la résistance est souvent associée à un faible rendement et à une qualité médiocre du grain chez le sorgho (Ramaiah et Parker 1982). Divers programmes ont donc été mis en place dans le but $d^{\prime}$ identifier des cultivars résistants et de transmettre cette résistance à des cultivars adaptés possédant des caractéristiques agronomiques et alimentaires intéressantes (Andrews 1970; King 1975; Obilana 1983; Ramaiah 1983).

Ces travaux ont permis d'identifier quelques cultivars de sorgho prometteurs. Parmi ceux-ci, le cultivar Framida est réputé pour son haut niveau de résistance au parasite et pour son rendement assez élevé et stable sous les conditions paysannes sahéliennes (Ramaiah 1984). Framida produit des plantules vigoureuses et possède une bonne résistance à la sécheresse, procurant ainsi une flexibilité au paysan quant au choix de la date du semis. II s'agit toutefois d'une variété à grains rouges, mal acceptée par les paysans à cause de ses piètres qualités gustatives et nutritives (ICRISAT 1983). Plusieurs essais ont été réalisés à I'ICRISAT (International Crops Research Institute for the Semi-Arid Tropics) au Burkina Faso dans le but de trouver à 'Framida' un dérivé à grains blancs présentant un potentiel de rendement élevé tout en conservant un haut niveau de résistance au $S$. hermonthica. Le processus de sélection des lignées a été décrit par Ramaiah (1987). Dans le cadre de notre recherche, nous avons évalué quelques-unes des lignées les plus prometteuses issues de ces travaux. Les critères d'évaluation comprenaient la résistance au $S$. hermonthica et le potentiel de rendement sous les conditions paysannes sahéliennes.

\section{MATÉRIEL ET MÉTHODES}

\section{Essais en pots}

Deux essais en pots ont été réalisés à la station expérimentale de Kamboinse, au Burkina Faso, en 1987. Le premier essai portait sur 20 lignées de sorgho de cycle court, incluant 19 lignées fixées ( $F_{6}$ et plus) issues du programme d'amélioration du sorgho de l'ICRISAT au Burkina Faso (ICSV1054 BF, ICSV-1055 BF, ICSV-1078 BF, ICSV-1079 BF, ICSV-1082 BF, ICSV-1083 BF, ICSV-1084 BF, ICSV-1085 BF, ICSV1086 BF, ICSV-1087 BF, ICSV-1099 BF, ICSV-1100 BF, ICSV-1101 BF, ICSV-1102 BF, ICSV-1103 BF, ICSV-1104 BF, ICSV$1105 \mathrm{BF}$, ICSV-1106 BF et ICSV-1107 BF) et un cultivar témoin sensible amélioré par I'IRAT (Institut de recherches agronomiques tropicales et des cultures vivrières) au Burkina Faso (IRAT-204). Le second essai portait sur 19 lignées de cycle moyen, incluant 18 lignées fixées ( $F_{6}$ et plus) issues du programme d'amélioration du sorgho de I'ICRISAT au Burkina Faso (ICSV1002 BF, ICSV-1036 BF, ICSV-1044 BF, ICSV-1049 BF, ICSV-1056 BF, ICSV-1063 BF, ICSV-1074 BF, ICSV-1088 BF, ICSV1089 BF, ICSV-1090 BF, ICSV-1091 BF, ICSV-1092 BF, ICSV-1093 BF, ICSV-1094 BF, ICSV-1095 BF, ICSV-1096 BF, ICSV1097 BF et ICSV-1098 BF) et un cultivar témoin sensible issu du programme d'amélioration du sorgho de I'ICRISAT en Inde (ICSV-126 IN). Les graines ont été fournies par I'ICRISAT au Burkina Faso. Un dispositif en blocs complets aléatoires comportant 10 répétitions a été utilisé dans chaque essai.

L'expérience a été menée à l'extérieur dans des pots de $22 \mathrm{~cm}$ de diam contenant un mélange de $2 \mathrm{~kg}$ de sol argileux, $1 \mathrm{~kg}$ de sable et $1 \mathrm{~kg}$ de fumier. Des graines de $S$. hermonthica (environ $150 \mathrm{mg}$ ) récoltées à Kamboinse en 1986 furent mélangées aux cinq premiers $\mathrm{cm}$ du sol de chaque pot avant le semis. Le sorgho a été semé le 21 mars à raison de cinq graines par pot. II a été démarié à deux, puis à un plant par pot, 1 et 2 semaines après le semis et 
irrigué chaque jour. Le nombre de plants de Striga émergés dans chaque pot fut déterminé 90 et 142 jours après le semis, respectivement, pour les essais sur les lignées de cycle court et de cycle moyen. La hauteur moyenne des trois plants de Striga les plus développés de chaque pot fut mesurée 123 jours après le semis. Les plants de Striga de chaque pot furent récoltés 156 et 152 jours après le semis, respectivement, pour les essais sur les lignées de cycles court et moyen, mis à sécher à $80^{\circ} \mathrm{C}$ pendant $48 \mathrm{~h}$ et pesés.

\section{Essais en champ infesté de Striga}

Deux essais ont été réalisés dans un champ infesté de $S$. hermonthica à Farako-ba, au Burkina Faso, en 1987. Le premier essai concernait les 20 lignées de cycle court testées en pots à Kamboinse. Le second portait sur les 19 lignées de cycle moyen testées en pots à Kamboinse, auxquelles était ajouté le témoin résistant 'Framida'. Un dispositif en blocs complets aléatoires comprenant quatre répétitions a été utilisé dans chaque essai. Des parcelles du témoin sensible 'CK-60B' ont été ajoutées systématiquement de façon à ce que chaque parcelle des lignées testées soit adjacente à une parcelle témoin disposée à sa gauche ou à sa droite dans un même bloc (Vasudeva Rao 1985).

Après le labour, un engrais fut appliqué à la volée aux doses de $21 \mathrm{~kg} \mathrm{ha}^{-1} \mathrm{~N}, 15 \mathrm{~kg}$ $\mathrm{ha}^{-1} \mathrm{P}$ et $19 \mathrm{~kg} \mathrm{ha}^{-1} \mathrm{~K}$. Un nivelage a précédé le semis. Chaque parcelle consistait en deux rangs de $4 \mathrm{~m}$, avec des écartements de $80 \mathrm{~cm}$ entre les rangs et de $40 \mathrm{~cm}$ entre les poquets d'un même rang. Cinq graines de sorgho ont été semées dans chaque poquet. Le semis a été effectué le 9 juillet pour les lignées de cycle court et le 8 juillet pour les lignées de cycle moyen. Les mauvaises herbes non parasites ont été sarclées à la main 1 semaine après le semis. Un démariage à deux plants par poquet a été effectué 24 jours après le semis, et de l'urée a été appliquée à la dose de $11,5 \mathrm{~kg} \mathrm{ha}^{-1} \mathrm{~N} 53$ jours après le semis. Le nombre de Striga émergés par parcelle a été déterminé 106 jours après le semis. Les panicules furent récoltées 119 jours après le semis, séchées au soleil et pesées, puis vannées pour permettre l'évaluation du poids des grains.

Essais en champ exempt de Striga

Deux essais ont été réalisés dans un champ exempt de $S$. hermonthica à Farako-ba, au Burkina Faso, en 1987. Le premier essai concernait les 20 lignées de cycle court testées en pots à Kamboinse et le second les 19 lignées de cycle moyen testées en pots à Kamboinse, auxquelles a été ajouté le témoin résistant 'Framida'. Un dispositif en bloc complet aléatoire comprenant quatre répétitions a été utilisé pour chaque essai.

Après le labour, on a appliqué à la volée $28 \mathrm{~kg} \mathrm{ha}^{-1} \mathrm{~N}, 20 \mathrm{~kg} \mathrm{ha}^{-1} \mathrm{P}$ et $25 \mathrm{~kg} \mathrm{ha}^{-1} \mathrm{~K}$. Un nivelage a précédé le semis. Chaque parcelle consistait en six rangs de $5 \mathrm{~m}$, avec des écartements de $80 \mathrm{~cm}$ entre les rangs et de $40 \mathrm{~cm}$ entre les poquets d'un même rang. Cinq graines de sorgho ont été semées dans chaque poquet. Les semis ont été réalisés les 10 et 3 juillet, respectivement, pour les essais sur les lignées de cycle court et de cycle moyen. Un démariage à trois plants par poquet fut effectué 21 jours après le semis. Les mauvaises herbes non parasites ont été sarclées à la main 19,33 et 68 jours après le semis dans les parcelles des lignées de cycle court et 13,34 et 75 jours après le semis dans les parcelles des lignées de cycle moyen. De l'urée a été appliquée en trois doses de $11,5 \mathrm{~kg} \mathrm{ha}^{-1} \mathrm{~N}$, et ce, 32,46 et 63 jours après le semis. Le nombre de jours depuis le semis jusqu'à $50 \%$ de floraison du sorgho a été noté dans chaque parcelle. Le nombre et la hauteur moyenne des plants de sorgho ont été évalués dans chaque parcelle 95 et 101 jours après le semis, respectivement, pour les essais sur les lignées de cycle court et de cycle moyen. Les panicules furent récoltées 113 et 115 jours après le semis, respectivement, séchées au soleil et pesées, puis vannées pour permettre la pesée des grains.

\section{Analyse statistique}

Les données ont été soumises à une analyse de variance, et les lignées ont été comparées à l'aide du test de comparaison multiple de Waller-Duncan (Waller et Duncan 1972). Les données ayant trait au nombre et à la biomasse du Striga ont été analysées après transformation logarithmique $[\log (x+1,1)]$. Les rendements en panicules et en grains du sorgho ont été analysés après transformation racinaire $(\sqrt{[x+0,5]})$. Les tableaux présentent les moyennes non transformées. Pour les essais en champ infesté, le nombre de 
Striga émergés dans la parcelle témoin adjacente à chacune des parcelles testées a été utilisé comme covariable pour l'analyse du nombre de Striga émergés (Bartlett 1978; Gilliver et al. 1985; Kempton et Howes 1981) à l'aide du système d'analyse statistique $\mathrm{SAS}^{\circledR}$ (Freund et al. 1986). Pour les essais en champ exempt de $S$. hermonthica, le nombre de plants de sorgho de chaque parcelle a été utilisé comme covariable pour l'analyse des données ayant trait aux rendements en panicules et en grains du sorgho.

\section{RÉSULTATS}

\section{Lignées de cycle court}

Dans l'essai effectué en pots, le nombre de Striga émergés sur les lignées ICSV$1079 \mathrm{BF}$ et ICSV-1078 BF a été inférieur $(P<0,0001)$ à celui de la plupart des autres lignées de l'essai (tableau 1). Le nombre de Striga émergés sur les lignées ICSV1082 BF et ICSV-1055 BF a aussi été significativement inférieur à celui de plusieurs des lignées de l'essai. Aucune autre lignée n'a subi une infestation du Striga inférieure à celle subie par le cultivar témoin sensible IRAT-204.

La biomasse aérienne sèche de Striga n'a été inférieure $(P=0,0342)$ à celle présente chez le témoin sensible IRAT-204 que chez les lignées ICSV-1101 BF, ICSV1078 BF, ICSV-1079 BF et ICSV-1083 BF (tableau 1). La hauteur moyenne des plants de Strigafut plus faible $(P<0,0001)$ pour les lignées ICSV-1079 BF et ICSV-1078 BF que pour la plupart des lignées de l'essai. Les lignées ICSV-1082 BF, ICSV-1055 BF, ICSV1054 BF, ICSV-1087 BF et ICSV-1106 BF ont supporté des plants de Striga dont la hauteur moyenne était inférieure à celle du Striga présent chez le témoin sensible IRAT-204.

Tableau 1. Nombre, biomasse sèche et hauteur moyenne des plants de Striga hermonthica sur 20 lignées de sorgho de cycle court cultivées en pots à Kamboinse au Burkina Faso en 1987

\begin{tabular}{lccc}
\hline $\begin{array}{l}\text { Lignée de } \\
\text { sorgho }\end{array}$ & $\begin{array}{c}\text { Nombre } \\
\text { de Striga } \\
\text { (nombre par pot) }^{\mathrm{a}}\end{array}$ & $\begin{array}{c}\text { Biomasse sèche } \\
\text { du Striga } \\
\text { (g par pot) }^{\mathrm{a}}\end{array}$ & $\begin{array}{c}\text { Hauteur } \\
\text { du Striga } \\
\text { (cm) }\end{array}$ \\
\hline ICSV-1079 BF & $3,0 \mathrm{a}^{\mathrm{b}}$ & $4,4 \mathrm{a}$ & $24,3 \mathrm{a}$ \\
ICSV-1078 BF & $3,9 \mathrm{a}$ & $4,2 \mathrm{a}$ & $26,7 \mathrm{a}$ \\
ICSV-1082 BF & $4,0 \mathrm{ab}$ & $3,1 \mathrm{abc}$ & $34,2 \mathrm{ab}$ \\
ICSV-1055 BF & $7,0 \mathrm{abc}$ & $4,9 \mathrm{abc}$ & $37,1 \mathrm{abc}$ \\
ICSV-1054 BF & $5,9 \mathrm{abcd}$ & $4,4 \mathrm{abc}$ & $37,7 \mathrm{abc}$ \\
ICSV-1101 BF & $7,1 \mathrm{bcde}$ & $3,3 \mathrm{a}$ & $46,2 \mathrm{bcdef}$ \\
ICSV-1087 BF & $8,4 \mathrm{cdef}$ & $4,3 \mathrm{abc}$ & $42,1 \mathrm{bcd}$ \\
ICSV-1083 BF & $9,6 \mathrm{cdef}$ & $3,2 \mathrm{ab}$ & $43,2 \mathrm{bcde}$ \\
ICSV-1106 BF & $9,8 \mathrm{cdef}$ & $3,7 \mathrm{abc}$ & $38,8 \mathrm{abc}$ \\
ICSV-1085 BF & $8,7 \mathrm{cdefg}$ & $3,3 \mathrm{abc}$ & $49,7 \mathrm{cdefg}$ \\
ICSV-1100 BF & $9,6 \mathrm{defgh}$ & $3,1 \mathrm{abc}$ & $53,6 \mathrm{defg}$ \\
ICSV-1086 BF & 9,5 efgh & $3,9 \mathrm{abc}$ & $50,3 \mathrm{cdefg}$ \\
ICSV-1099 BF & 9,9 efgh & $4,2 \mathrm{abc}$ & $56,5 \mathrm{defg}$ \\
ICSV-1105 BF & 10,5 efgh & $8,1 \mathrm{abc}$ & $57,3 \mathrm{efg}$ \\
ICSV-1102 BF & $11,5 \mathrm{fgh}$ & $5,0 \mathrm{abc}$ & $55,9 \mathrm{defg}$ \\
ICSV-1107 BF & $12,4 \mathrm{fgh}$ & $7,3 \mathrm{bc}$ & $59,7 \mathrm{fg}$ \\
ICSV-1084 BF & $13,3 \mathrm{fgh}$ & $6,2 \mathrm{abc}$ & $59,2 \mathrm{fg}$ \\
ICSV-1103 BF & $15,5 \mathrm{gh}$ & $3,6 \mathrm{abc}$ & $54,0 \mathrm{defg}$ \\
ICSV-1104 BF & $16,2 \mathrm{~h}$ & $7,1 \mathrm{abc}$ & $61,9 \mathrm{~g}$ \\
IRAT-204 & 9,1 defgh & $7,9 \mathrm{c}$ & $57,4 \mathrm{efg}$ \\
Moyenne & 9,2 & 4,8 & 47,0 \\
Erreur type & 1,6 & 1,3 & 5,2 \\
\hline
\end{tabular}

\footnotetext{
a L'analyse a été exécutée après transformation logarithmique des données $(\log [x+1,1])$.

b Les moyennes suivies de la même lettre ne diffèrent pas significativement selon le test de WallerDuncan.
} 
Tableau 2. Nombre de plants de Striga hermonthica et rendement en panicules et en grains de 21 lignées de sorgho de cycle court cultivées en champ infesté de Striga à Farako-ba au Burkina Faso en 1987

\begin{tabular}{lccc}
\hline $\begin{array}{l}\text { Lignée de } \\
\text { sorgho }\end{array}$ & $\begin{array}{c}\text { Nombre } \\
\text { de Striga } \\
(\text { nombre m }\end{array}$ & $\begin{array}{c}\text { Rendement en } \\
\text { panicules } \\
\left(\mathrm{kg} \mathrm{ha}^{-1}\right)^{\mathrm{b}}\end{array}$ & $\begin{array}{c}\text { Rendement } \\
\text { en grains } \\
\left(\mathrm{kg} \mathrm{ha}^{-1}\right)^{\mathrm{b}}\end{array}$ \\
\hline ICSV-1079 BF & $3,5 \mathrm{bc}^{\mathrm{c}}$ & $1551 \mathrm{ab}$ & $1100 \mathrm{abc}$ \\
ICSV-1078 BF & $3,9 \mathrm{abc}$ & $1135 \mathrm{abc}$ & $863 \mathrm{abcde}$ \\
ICSV-1082 BF & $2,8 \mathrm{abc}$ & $1338 \mathrm{abc}$ & $953 \mathrm{abcd}$ \\
ICSV-1055 BF & $5,5 \mathrm{abc}$ & $1139 \mathrm{abc}$ & $734 \mathrm{abcde}$ \\
ICSV-1054 BF & $5,8 \mathrm{abc}$ & $1262 \mathrm{abc}$ & $945 \mathrm{abcde}$ \\
ICSV-1101 BF & $3,5 \mathrm{abc}$ & $772 \mathrm{bc}$ & $539 \mathrm{cde}$ \\
ICSV-1087 BF & $5,1 \mathrm{a}$ & $758 \mathrm{bc}$ & $600 \mathrm{bcde}$ \\
ICSV-1083 BF & $8,2 \mathrm{abc}$ & $1332 \mathrm{abc}$ & $988 \mathrm{abcde}$ \\
ICSV-1106 BF & $6,8 \mathrm{abc}$ & $1270 \mathrm{abc}$ & $934 \mathrm{abcde}$ \\
ICSV-1085 BF & $11,7 \mathrm{abc}$ & $920 \mathrm{bc}$ & $492 \mathrm{cde}$ \\
ICSV-1100 BF & $8,4 \mathrm{c}$ & $1022 \mathrm{bc}$ & $682 \mathrm{abcde}$ \\
ICSV-1086 BF & $7,9 \mathrm{abc}$ & $844 \mathrm{bc}$ & $545 \mathrm{bcde}$ \\
ICSV-1099 BF & $5,4 \mathrm{bc}$ & $1443 \mathrm{ab}$ & $1091 \mathrm{ab}$ \\
ICSV-1105 BF & $12,8 \mathrm{abc}$ & $2055 \mathrm{a}$ & $1342 \mathrm{a}$ \\
ICSV-1102 BF & $15,1 \mathrm{c}$ & $1147 \mathrm{abc}$ & $815 \mathrm{abcde}$ \\
ICSV-1107 BF & $5,0 \mathrm{abc}$ & $1313 \mathrm{abc}$ & $830 \mathrm{abcde}$ \\
ICSV-1084 BF & $11,2 \mathrm{c}$ & $813 \mathrm{bc}$ & $449 \mathrm{de}$ \\
ICSV-1103 BF & $2,9 \mathrm{abc}$ & $615 \mathrm{c}$ & $416 \mathrm{e}$ \\
ICSV-1104 BF & $10,5 \mathrm{c}$ & $830 \mathrm{bc}$ & $533 \mathrm{bcde}$ \\
& & & $611 \mathrm{bcde}$ \\
IRAT-204 & $3,0 \mathrm{ab}$ & $910 \mathrm{bc}$ & 368 \\
CK-60B & 13,4 & 603 & 763 \\
Moyenne & 7,0 & 1124 & 183 \\
Erreur type & 4,2 & 240 & \\
\hline
\end{tabular}

${ }^{a} L$ 'analyse a été exécutée après transformation logarithmique des données $(\log [x+1,1])$.

${ }^{b}$ L'analyse a été exécutée après transformation racine carrée des données $(\sqrt{[x+0,5]})$.

c Les moyennes suivies de la même lettre ne diffèrent pas significativement selon le test de WallerDuncan.

Dans l'essai en champ infesté, le nombre de Striga émergés sur la lignée ICSV$1087 \mathrm{BF}$ était inférieur $(P=0,0393)$ à celui de six des lignées de l'expérience (tableau 2). Aucune lignée $n^{\prime}$ a subi une infestation significativement inférieure à celle subie par le témoin sensible IRAT-204.

Toutes les lignées ont obtenu un faible rendement en conditions infestées. Chez la lignée ICSV-1105 BF, le poids des panicules a cependant été supérieur $(P=0,0165)$ à celui de plusieurs des lignées de l'expérience (tableau 2). La lignée ICSV-1105 $\mathrm{BF}$ a également obtenu un rendement en grains supérieur $(P=0,0092)$ à celui de plusieurs lignées, soit $1342 \mathrm{~kg} \mathrm{ha}^{-1}$, comparativement à $763 \mathrm{~kg} \mathrm{ha}^{-1}$ pour la moyenne de toutes les lignées de l'essai.
En l'absence de Striga, les lignées ICSV1105 BF et ICSV-1103 BF ont obtenu un rendement en panicules supérieur $(P=0,0124)$ à celui de plusieurs des lignées de l'essai (tableau 3). La lignée ICSV-1104 $\mathrm{BF}$ a aussi obtenu un rendement en panicules élevé.

Le rendement en grains de ICSV -1103 $\mathrm{BF}$ a été supérieur $(P=0,0184)$ à celui de quelques-unes des lignées de l'essai, soit $2837 \mathrm{~kg} \mathrm{ha}^{-1}$, comparativement à $2228 \mathrm{~kg}$ $\mathrm{ha}^{-1}$ pour la moyenne des lignées. ICSV$1105 \mathrm{BF}$ a aussi obtenu un rendement en grains élevé (tableau 3). La date de floraison et la hauteur de chacune des lignées de sorgho sont rapportées dans le tableau 3. 


\section{Lignées de cycle moyen}

Dans l'essai mené en pots, le nombre de Striga émergés sur les lignées ICSV-1074 $B F$ et ICSV-1090 BF a été inférieur $(P=0,0012)$ à celui observé sur quelquesunes des autres lignées de l'essai (tableau 4). Aucune lignée n'a subi une infestation inférieure à celle subie par le cultivar témoin sensible ICSV-126 IN, y compris le cultivar réputé modérément résistant ICSV-1002 BF (Ramaiah 1986).

La biomasse aérienne sèche de Striga n'était inférieure $(P=0,0052)$ à celle présente chez le témoin sensible ICSV-126 IN que chez la lignée ICSV-1044 BF (tableau 4). La hauteur moyenne des plants de Striga a été élevée pour toutes les lignées de l'essai, avec une moyenne de $44,9 \mathrm{~cm}$.
Dans l'essai effectué en champ infesté, le nombre de Striga émergés sur la lignée ICSV-1089 BF, soit 0,7 plant $\mathrm{m}^{-2}$ (tableau 5), était inférieur $(P=0,0466)$ à celui présent chez plusieurs des lignées de l'essai, y compris le témoin résistant 'Framida'.

La lignée ICSV-1096 BF a obtenu un rendement en panicules inférieur $(P=0,0328)$ à celui de plusieurs des lignées de l'expérience (tableau 5). Toutes les autres lignées ont obtenu des rendements en panicules qui n'étaient pas différents les uns des autres. La lignée ICSV-1002 BF a obtenu un rendement en grains supérieur $(P=0,0135)$ à celui de trois lignées seulement, avec $1223 \mathrm{~kg} \mathrm{ha}^{-1}$ en moyenne, comparativement à $869 \mathrm{~kg} \mathrm{ha}^{-1}$ pour la moyenne de toutes les lignées de l'essai.

Tableau 3. Rendement en panicules et en grains, date de floraison et hauteur de 20 lignées de sorgho de cycle court cultivées en champ exempt de Striga hermonthica à Farako-ba au Burkina Faso en 1987

\begin{tabular}{|c|c|c|c|c|}
\hline $\begin{array}{l}\text { Lignée de } \\
\text { sorgho }\end{array}$ & $\begin{array}{c}\text { Rendement } \\
\text { en panicules } \\
\left(\mathrm{kg} \mathrm{ha}^{-1}\right)^{\mathrm{a}}\end{array}$ & $\begin{array}{l}\text { Rendement } \\
\text { en grains } \\
\left(\mathrm{kg} \mathrm{ha}^{-1}\right)^{\mathrm{a}}\end{array}$ & $\begin{array}{l}\text { Nombre de jours } \\
\text { jusqu'à la } \\
\text { floraison de } 50 \% \\
\text { du sorgho }\end{array}$ & $\begin{array}{l}\text { Hauteur } \\
\text { du } \\
\text { sorgho } \\
\text { (cm) }\end{array}$ \\
\hline ICSV-1079 BF & $2696 \mathrm{def}^{\mathrm{b}}$ & 2026 abcd & $69,0 \mathrm{ghi}$ & $171,3 \mathrm{fg}$ \\
\hline ICSV-1078 BF & 3223 abcdef & $2457 \mathrm{abc}$ & $65,5 \mathrm{i}$ & $182,5 \mathrm{efg}$ \\
\hline ICSV-1082 BF & 2663 cdef & $1823 \mathrm{~cd}$ & 72,8 cdefg & $171,3 \mathrm{fg}$ \\
\hline ICSV-1055 BF & 2676 def & 1984 bcd & $69,5 \mathrm{fghi}$ & $168,8 \mathrm{gh}$ \\
\hline ICSV-1054 BF & 2858 bcdef & $2041 \mathrm{abcd}$ & $66,0 \mathrm{i}$ & $181,3 \mathrm{efg}$ \\
\hline ICSV-1101 BF & $3573 \mathrm{abcd}$ & $2438 a b c$ & 73,3 bcdef & 196,3 bcdef \\
\hline ICSV-1087 BF & 2766 cdef & $1931 \mathrm{abcd}$ & $68,0 \mathrm{hi}$ & $145,0 \mathrm{hi}$ \\
\hline ICSV-1083 BF & 3206 abcdef & $2242 \mathrm{abcd}$ & 75,8 abcd & $213,8 \mathrm{abc}$ \\
\hline ICSV-1106 BF & 3556 abcd & $2601 \mathrm{abc}$ & $75,3 \mathrm{abcd}$ & $211,3 \mathrm{abcd}$ \\
\hline ICSV-1085 BF & $2306 \mathrm{f}$ & $1475 \mathrm{~d}$ & $78,8 \mathrm{a}$ & 197,5 bcde \\
\hline ICSV-1100 BF & 3466 abcde & 2378 abcd & $76,3 \mathrm{abc}$ & 201,3 bcde \\
\hline ICSV-1086 BF & 2779 cdef & $1700 \mathrm{~cd}$ & 73,8 bcde & 186,3 defg \\
\hline ICSV-1099 BF & 2598 ef & $1852 \mathrm{~cd}$ & 71,8 defgh & 190,0 cdefg \\
\hline ICSV-1105 BF & $4172 \mathrm{a}$ & $2763 a b$ & 70,5 efgh & 187,5 defg \\
\hline ICSV-1102 BF & 3538 abcde & $2495 a b c$ & $77,3 \mathrm{ab}$ & $213,8 \mathrm{abc}$ \\
\hline ICSV-1107 BF & 3326 abcde & $2106 \mathrm{abcd}$ & 70,8 efgh & $171,3 \mathrm{fg}$ \\
\hline ICSV-1084 BF & 3551 abcde & $2378 a b c$ & $76,5 \mathrm{abc}$ & $221,3 a b$ \\
\hline ICSV-1103 BF & $3892 a b$ & 2837 a & $69,3 \mathrm{fghi}$ & 202,5 abcde \\
\hline ICSV-1104 BF & 3752 abc & $2454 a b c$ & 72,8 cdefg & $227,5 \mathrm{a}$ \\
\hline IRAT-204 & $3593 \mathrm{abcd}$ & $2577 \mathrm{abc}$ & $61,0 \mathrm{j}$ & $131,3 i$ \\
\hline Moyenne & 3210 & 2228 & 71,7 & 188,6 \\
\hline Erreur type & 396 & 312 & 1,6 & 9,4 \\
\hline
\end{tabular}

a L'analyse a été exécutée après transformation racine carrée des données $(\sqrt{[x+0,5]})$. Le nombre de plants de sorgho de chaque parcelle a été utilisé comme covariable.

b Les moyennes suivies de la même lettre ne diffèrent pas significativement selon le test de WallerDuncan. 
Tableau 4. Nombre, biomasse sèche et hauteur moyenne des plants de Striga hermonthica sur 19 lignées de sorgho de cycle moyen cultivées en pots à Kamboinse au Burkina Faso en 1987

\begin{tabular}{|c|c|c|c|}
\hline $\begin{array}{l}\text { Lignée de } \\
\text { sorgho }\end{array}$ & $\begin{array}{c}\text { Nombre } \\
\text { de Striga } \\
\text { (nombre par pot) }^{a}\end{array}$ & $\begin{array}{c}\text { Biomasse sèche } \\
\text { de Striga } \\
(\mathrm{g} \text { par pot })^{a}\end{array}$ & $\begin{array}{l}\text { Hauteur } \\
\text { du Striga } \\
\text { (cm) }\end{array}$ \\
\hline ICSV-1074 BF & $6,4 a^{b}$ & $8,8 \mathrm{de}$ & 43,4 \\
\hline ICSV-1090 BF & $6,9 \mathrm{a}$ & $4,1 \mathrm{ab}$ & 43,2 \\
\hline ICSV-1089 BF & $6,7 \mathrm{ab}$ & 5,1 abcde & 50,4 \\
\hline ICSV-1093 BF & 7,3 abc & 6,2 bcde & 44,7 \\
\hline ICSV-1096 BF & $7,9 \mathrm{abc}$ & 8,1 bcde & 48,9 \\
\hline ICSV-1044 BF & 8,9 abc & $3,3 a$ & 34,4 \\
\hline ICSV-1056 BF & 7,9 abcd & 7,6 cde & 44,7 \\
\hline ICSV-1098 BF & 8,4 abcd & 5,7 bcde & 50,5 \\
\hline ICSV-1036 BF & 8,8 abcd & 5,9 abcde & 47,4 \\
\hline ICSV-1002 BF & 9,0 abcd & $5,2 \mathrm{abcd}$ & 38,6 \\
\hline ICSV-1049 BF & 9,3 abcd & $4,6 a b c$ & 43,1 \\
\hline ICSV-1091 BF & 9,9 abcd & 6,3 abcde & 43,9 \\
\hline ICSV-1063 BF & 10,0 abcde & $11,5 \mathrm{e}$ & 44,2 \\
\hline ICSV-1092 BF & 12,7 bcde & 6,7 bcde & 46,1 \\
\hline ICSV-1097 BF & $11,9 \mathrm{cde}$ & 6,5 bcde & 47,7 \\
\hline ICSV-1094 BF & $12,9 \mathrm{de}$ & 5,2 abcde & 45,3 \\
\hline ICSV-1095 BF & 13,4 de & 6,6 abcde & 48,7 \\
\hline ICSV-1088 BF & $16,2 \mathrm{e}$ & 9,0 bcde & 47,4 \\
\hline ICSV-126 IN & 11,3 abcde & 7,3 bcde & 41,6 \\
\hline Moyenne & 9,8 & 6,5 & 44,9 \\
\hline Erreur type & 1,6 & 1,7 & 2,6 \\
\hline
\end{tabular}

a L'analyse a été exécutée après transformation logarithmique des données (log[x+1,1]).

${ }^{b}$ Les moyennes suivies de la même lettre ne diffèrent pas significativement selon le test de WallerDuncan.

Dans l'essai mené en champ exempt de Striga, les lignées ICSV-1056 BF et ICSV$1063 \mathrm{BF}$ ont obtenu un rendement en panicules supérieur $(P=0,0021)$ à celui de quelques-unes des lignées de l'essai (tableau 6). Le rendement en grains de la lignée ICSV-1056 BF était supérieur $(P=0,0002)$ à celui de plusieurs lignées, avec $3325 \mathrm{~kg} \mathrm{ha}^{-1}$ en moyenne, comparativement à $2517 \mathrm{~kg} \mathrm{ha}^{-1}$ pour la moyenne des lignées de l'essai. ICSV-1063 BF a aussi obtenu un rendement en grains élevé. Aucune lignée n'a obtenu un rendement supérieur à celui de 'Framida'. La date de floraison et la hauteur de chacune des lignées de sorgho sont données dans le tableau 6.

\section{DISCUSSION}

Dans l'essai en pots sur les lignées de cycle court, les lignées ICSV-1078 BF et ICSV-1079 BF ont subi une infestation plus faible que la plupart des autres lignées. Cette faible émergence du parasite était associée à une croissance réduite des plants de Striga. Une faible émergence de Striga a aussi été observée chez la lignée ICSV-1082 BF. Ces trois lignées ont malgré tout montré une sensibilité relativement élevée, ne présentant jamais moins de trois plants par pot en moyenne, comparativement à 9,1 plants par pot pour le témoin sensible IRAT-204. Cette sensibilité apparente a été confirmée au champ. Les lignées ICSV-1078 BF, ICSV-1079 BF et ICSV-1082 BF n'ont pas pu se démarquer des autres de façon significative. En fait, aucune lignée n'a présenté une sensibilité inférieure à celle du témoin sensible IRAT204. Presque toutes les autres lignées ont obtenu de faibles rendements au champ. Seule la lignée ICSV-1105 BF a produit un rendement adéquat en champinfesté, soit $1342 \mathrm{~kg} \mathrm{ha}^{-1}$. Elle est toutefois apparue fortement attaquée par le $S$. hermonthica. Sans être résistante, cette lignée semble 
donc posséder un bon niveau de tolérance au Striga.

Dans l'essai en pots sur les lignées de cycle moyen, les lignées ICSV-1074 BF et ICSV-1090 BF ont subi une infestation inférieure à celle subie par quelques-unes des autres lignées. Aucune lignée n'a cependant subi une infestation inférieure à celle du témoin sensible ICSV-126 IN, et toutes ont présenté plus de six plants de Striga par pot en moyenne. Même le cultivar ICSV-1002 BF, qui est réputé pour son niveau de résistance modéré (Ramaiah 1986), est apparu très sensible dans cet essai. Ces caractéristiques ont généralement été confirmées au champ. Seule la lignée ICSV-1089 BF a montré une sensi- bilité plutôt faible et inférieure à celle de plusieurs des lignées, y compris le témoin résistant 'Framida' et le cultivar ICSV-1002 BF. Ces deux cultivars, bien qu'ils aient obtenu d'assez bons rendements en champ infesté, ont supporté de fortes infestations.

Toutes les lignées de cycles court et moyen se sont donc avérées sensibles, et cela aussi bien en pots qu'au champ. Seule ICSV-1089 BF est apparue peu sensible au champ, mais non en pots, ce qui pourrait indiquer que son potentiel de résistance est faible en présence de fortes infestations. De plus, cette lignée s'est caractérisée par des rendements médiocres au champ. Elle ne saurait donc être

Tableau 5. Nombre de plants de Striga hermonthica et rendement en panicules et en grains de 21 lignées de sorgho de cycle moyen cultivées en champ infesté de Striga à Farako-ba au Burkina Faso en 1987

\begin{tabular}{|c|c|c|c|}
\hline $\begin{array}{l}\text { Lignée de } \\
\text { sorgho }\end{array}$ & $\begin{array}{c}\text { Nombre } \\
\text { de Striga } \\
\left(\text { nombre } \mathrm{m}^{-2}\right)^{a}\end{array}$ & $\begin{array}{c}\text { Rendement en } \\
\text { panicules } \\
\left(\mathrm{kg} \mathrm{ha}^{-1}\right)^{\mathrm{b}}\end{array}$ & $\begin{array}{l}\text { Rendement } \\
\text { en grains } \\
\left(\mathrm{kg} \mathrm{ha}^{-1}\right)^{\mathrm{b}}\end{array}$ \\
\hline ICSV-1074 BF & $6,6 \mathrm{abcd}^{\mathrm{c}}$ & $1121 \mathrm{ab}$ & $834 a b$ \\
\hline ICSV-1090 BF & 5,8 abcd & $1281 \mathrm{a}$ & $841 \mathrm{ab}$ \\
\hline ICSV-1089 BF & $0,7 \mathrm{a}$ & $1307 \mathrm{a}$ & $928 \mathrm{ab}$ \\
\hline ICSV-1093 BF & $2,0 a b$ & 1418 a & $994 a b$ \\
\hline ICSV-1096 BF & $18,3 \mathrm{bcd}$ & $529 b$ & $383 \mathrm{c}$ \\
\hline ICSV-1044 BF & $12,4 \mathrm{abc}$ & 1578 a & $1154 a b$ \\
\hline ICSV-1056 BF & $9,8 \mathrm{bcd}$ & $856 a b$ & 516 bc \\
\hline ICSV-1098 BF & $8,7 \mathrm{ab}$ & $947 a b$ & 598 abc \\
\hline ICSV-1036 BF & $11,2 \mathrm{abc}$ & $1264 \mathrm{a}$ & $951 \mathrm{ab}$ \\
\hline ICSV-1002 BF & 9,8 bcd & 1635 a & $1223 \mathrm{a}$ \\
\hline ICSV-1049 BF & 2,4 abc & 1316 a & $910 a b$ \\
\hline ICSV-1091 BF & $10,0 \mathrm{abc}$ & 1566 a & $1068 a b$ \\
\hline ICSV-1063 BF & $15,0 \mathrm{bcd}$ & 1502 a & $1059 a b$ \\
\hline ICSV-1092 BF & $10,7 \mathrm{bcd}$ & 1426 a & $1020 a b$ \\
\hline ICSV-1097 BF & $23,2 \mathrm{~d}$ & $815 a b$ & 522 bc \\
\hline ICSV-1094 BF & $18,4 \mathrm{~cd}$ & 1408 a & $979 a b$ \\
\hline ICSV-1095 BF & $15,7 \mathrm{~cd}$ & $936 a b$ & $566 \mathrm{abc}$ \\
\hline ICSV-1088 BF & $2,9 a b c$ & 1418 a & $984 a b$ \\
\hline ICSV-126 IN & $17,1 \mathrm{bcd}$ & $1084 a b$ & $731 \mathrm{abc}$ \\
\hline Framida & $13,5 \mathrm{bcd}$ & 1520 a & $1119 a b$ \\
\hline CK-60B & 15,5 & 459 & 275 \\
\hline Moyenne & 10,7 & 1246 & 869 \\
\hline Erreur type & 7,2 & 245 & 180 \\
\hline
\end{tabular}

a L'analyse a été exécutée après transformation logarithmique des données $(\log [x+1,1])$.

${ }^{b}$ L'analyse a été exécutée après transformation racine carrée des données $(\sqrt{[x+0,5]})$.

c Les moyennes suivies de la même lettre ne diffèrent pas significativement selon le test de W/allerDuncan. 
Tableau 6. Rendement en panicules et en grains, date de floraison et hauteur de 20 lignées de sorgho de cycle moyen cultivées en champ exempt de Striga hermonthica à Farako-ba au Burkina Faso en 1987

\begin{tabular}{|c|c|c|c|c|}
\hline $\begin{array}{l}\text { Lignée de } \\
\text { sorgho }\end{array}$ & $\begin{array}{c}\text { Rendement } \\
\text { en panicules } \\
\left(\mathrm{kg} \mathrm{ha}^{-1}\right)^{\mathrm{a}}\end{array}$ & $\begin{array}{l}\text { Rendement } \\
\text { en grains } \\
\left(\mathrm{kg} \mathrm{ha}^{-1}\right)^{\mathrm{a}}\end{array}$ & $\begin{array}{l}\text { Nombre de jours } \\
\text { jusqu'à la } \\
\text { floraison de } 50 \% \\
\text { du sorgho }\end{array}$ & $\begin{array}{l}\text { Hauteur } \\
\text { du } \\
\text { sorgho } \\
\text { (cm) }\end{array}$ \\
\hline ICSV-1074 BF & $3499 b c^{b}$ & 2576 abcde & $70,8 \mathrm{bc}$ & 225,0 cdef \\
\hline ICSV-1090 BF & $3948 \mathrm{abc}$ & 2567 abcde & 67,8 defg & $242,5 \mathrm{abcd}$ \\
\hline ICSV-1089 BF & 3560 bc & 2498 bcde & $74,3 \mathrm{a}$ & 223,8 cdef \\
\hline ICSV-1093 BF & $3752 \mathrm{abc}$ & 2670 abcde & 69,5 cde & 275,0 a \\
\hline ICSV-1096 BF & $3748 \mathrm{abc}$ & 2717 abcd & $70,8 \mathrm{bc}$ & 231,3 bcdef \\
\hline ICSV-1044 BF & $3156 \mathrm{~cd}$ & 2175 de & 68,8 cdefg & 197,5 efg \\
\hline ICSV-1056 BF & 4693 a & 3325 a & 69,0 cdef & $248,8 \mathrm{abc}$ \\
\hline ICSV-1098 BF & $3748 a b c$ & 2560 abcde & 70,8 bc & 218,8 cdef \\
\hline ICSV-1036 BF & $3872 \mathrm{abc}$ & 2773 abcd & 69,3 cdef & 205,0 defg \\
\hline ICSV-1002 BF & $3706 \mathrm{abc}$ & 2727 abcd & 68,8 cdefg & 223,8 cdef \\
\hline ICSV-1049 BF & 3652 bc & 2531 bcde & 70,0 cde & $192,5 \mathrm{fg}$ \\
\hline ICSV-1091 BF & 3956 abc & 2441 bcde & $66,5 \mathrm{fg}$ & $267,5 a b$ \\
\hline ICSV-1063 BF & $4374 a b$ & $3146 a b$ & 68,3 cdefg & 236,3 abcde \\
\hline ICSV-1092 BF & 3499 bc & 2313 cde & $73,8 \mathrm{a}$ & 216,3 cdef \\
\hline ICSV-1097 BF & $2476 d$ & $1449 \mathrm{f}$ & $73,0 a b$ & $171,3 \mathrm{gh}$ \\
\hline ICSV-1094 BF & 3089 cd & 1991 ef & $66,0 \mathrm{~g}$ & $241,3 \mathrm{abcd}$ \\
\hline ICSV-1095 BF & 3639 abc & $2131 \mathrm{de}$ & 70,5 bcd & 240,0 abcd \\
\hline ICSV-1088 BF & 3634 bc & 2371 cde & 68,0 cdefg & $142,5 \mathrm{~h}$ \\
\hline ICSV-126 IN & 3377 c & 2395 cde & 74,8 a & 206,3 defg \\
\hline Framida & $3960 \mathrm{abc}$ & $3015 \mathrm{abc}$ & 67,5 efg & $241,3 \mathrm{abcd}$ \\
\hline Moyenne & 3668 & 2517 & 69,9 & 222,3 \\
\hline Erreur type & 314 & 252 & 1,0 & 14,2 \\
\hline
\end{tabular}

a L'analyse a été exécutée après transformation racine carrée des données $(\sqrt{[x+0,5]})$. Le nombre de plants de sorgho de chaque parcelle a été utilisé comme covariable.

${ }^{b}$ Les moyennes suivies de la même lettre ne diffèrent pas significativement selon le test de WallerDuncan.

recommandée. Bien que certaines lignées aient un fort potentiel de rendement en l'absence de Striga, toutes sont apparues sensibles et peu adaptées. Puisque les conditions de régie (faibles apports d'intrants) utilisées lors des expériences menées au champ correspondent à celles utilisées par les paysans sahéliens, aucune des lignées testées ne peut donc être recommandée pour la résistance au $S$. hermonthica.

De façon surprenante, le cultivar Framida, qui a été utilisé pour tenter de transmettre sa résistance à d'autres cultivars, est apparu modérément sensible à I'attaque par le $S$. hermonthica. L'utilisation de 'Framida' ne saurait donc empêcher la constitution d'une banque de graines de $S$. hermonthica dans le sol. II semblerait parconséquent plus juste, dans le cas de ce cultivar, de parler de tolérance au S. hermonthica plutôt que de résistance, à moins que la résistance de ce cultivar ait été instable et rapidement perdue.

La sensibilité modérée de 'Framida' ne signifie pas que ce cultivar ne possède aucun mécanisme de résistance. II a été démontré que 'Framida' stimule faiblement la germination du Striga (Williams 1959). De même, Maiti et al. (1984) ont rapporté que ce cultivar pourrait avoir une résistance constitutive basée sur la subérisation des parois des cellules de l'endoderme, une forte lignification du péricycle, et la présence de dépôts de silice. 'Framida' présente aussi un certain intérêt à cause de son bon potentiel de rendement et de la stabilité de ce rendement 
aux niveaux d'intrants minimaux (Matlon 1985). La tolérance au Striga, même si elle ne peut empêcher l'expansion des infestations, demeure une caractéristique souhaitable en l'absence de cultivars résistants adaptés aux conditions sahéliennes. Cependant, la résistance de 'Framida' ne semble pas suffisante pour supporter un programme d'amélioration génétique du sorgho pour la résistance au $S$. hermonthica. Aucune des lignées testées, qui furent pour leur grande majorité créées en utilisant 'Framida' comme géniteur résistant, ne s'est avérée résistante au parasite. On peut alors se demander si le choix de ce géniteur était bien approprié. Ramaiah (1984) a rapporté que 'Framida' n'était pas un bon combinant général pour la résistance au Striga. II est donc probable qu'il faille aller chercher ailleurs que chez 'Framida' la source de la résistance au $S$. hermonthica chez le sorgho.

En l'absence de cultivar hautement résistant adapté aux conditions sahéliennes, le paysan $n^{\prime}$ a présentement pas d'autre choix que d'utiliser le cultivar local amélioré possédant le plus haut niveau de tolérance possible à l'attaque du $S$. hermonthica. Dans ce contexte, il apparaît toujours de plus en plus urgent d'identifier des sources de haute résistance au S. hermonthica chez le sorgho. C'est peut-être sur la base de mécanismes de résistance connus que devrait se faire la sélection du sorgho à l'avenir. L'existence de quelques centaines de lignées de sorgho reconnues pour leur faible production de stimulants de la germination de $S$. asiatica en Inde a déjà été rapportée (ICRISAT 1981). L'identification parmi celles-ci d'un ou de quelques cultivars possédant un haut niveau de résistance au $S$. hermonthica en Afrique de l'Ouest (Olivier et al. 1991) pourrait permettre d'entreprendre des travaux d'amélioration génétique du sorgho sur la base d'un mécanisme de résistance connu, d'autant plus que l'héritabilité du caractère de faible production de stimulants de germination a été établie (Ramaiah et al. 1990).

Il semble bien que le programme d'amélioration du sorgho pour la résistance au S. hermonthica au Burkina Faso se soit soldé par un échec relatif. Toutes les lignées, y compris 'Framida', sont sensibles, même si elles possèdent souvent des caractéristiques agronomiques intéressantes. Le cultivar Framida ne saurait être utilisé comme source de résistance à l'intérieur d'un programme d'amélioration génétique du sorgho. La nécessité de trouver d'autres sources de résistance se fait donc pressante. II est important de mieux comprendre les mécanismes impliqués dans la résistance du sorgho au $S$. hermonthica pour pouvoir effectuer une sélection variétale sur la base des mécanismes de résistance.

\section{REMERCIEMENTS}

Ce travail a été rendu possible grâce au support financier du Centre Sahel de I'Université Laval et de l'Agence canadienne de développement international. Le premier auteur est boursier du Conseil de la recherche en sciences naturelles et génie du Canada. Nous tenons à remercier Michèle Martel pour son assistance technique soutenue, Paul Kaboré et Anselme Conombo pour leur aide technique dans l'expérimentation au champ et JeanMarc Girard pour ses conseils lors de I'analyse statistique des données.

\section{RÉFÉRENCES}

Andrews, D.J. 1970. Breeding and testing dwarf sorghums in Nigeria. Expl. Agric. 6: 41-50.

Bartlett, M.S. 1978. Nearest neighbour models in the analysis of field experiments. J. Royal Stat. Soc. B 40: 147-174.

Doggett, H. 1952. Annual Report of the Botanist, Ukiriguru, for the year 1950. Pages 222-244 in Annual Report 1950, Tanganyika Department of Agriculture.

Doggett, H. 1965. Striga hermonthica on sorghum in East Africa. J. Agric. Sci. 65: 183194.

Doggett, H. 1988. Sorghum. Tropical Agriculture Series, 2nd ed., Longman Scientific and Technical-IDRC, New York. p. 3/3.

Freund, R.J., R.C. Littlell et P.C. Spector. 1986. SAS system for linear models. SAS Series in Statistical Applications, SAS Institute Inc., Cary, N.C. p. 142-147.

Gilliver, B., M.J. Vasudeva Rao et P. Venkateswarlu. 1985. A design and methods to monitor crop growth conditions illustrated with sorghum screening trials for resistance to Striga. Expl. Agric. 21: 233-240.

ICRISAT. 1981. Annual Report 1979/80. Patancheru, India: ICRISAT, p. 22-23.

ICRISAT. 1983. Annual Report 1982. Patancheru, India: ICRISAT, p. 344 
Kempton, R.A. et C.W. Howes. 1981. The use of neighbouring plot values in the analysis of variety trials. Appl. Stat. 30: 59-70.

King, S.B. 1975. Screening sorghum for resistance to witchweed Striga hermonthica in Nigeria. Page 136 in Proc. Am. Phytopathol. Soc. Southern Division, vol. 2, 2-5 Feb. 1975, U.S.A.

Maiti, R.K., K.V. Ramaiah, S.S. Bisen et V.L. Chidley. 1984. A comparative study of the haustorial development of Striga asiatica (L.) Kuntze on sorghum cultivars. Ann. Bot. 54: 447-457.

Matlon, P.J. 1985. Analyse critique des objectifs, méthodes et progrès accomplis à ce jour dans l'amélioration du sorgho et du mil : une étude de cas de l'ICRISAT/Burkina Faso. Pages 181-211 in H.W. Ohm et J.G. Nagy (réd.), Technologies appropriées pour les paysans des zones semi-arides de l'Afrique de I'Ouest. Université de Purdue, U.S.A.

Obilana, A.T. 1983. Striga studies and control in Nigeria. Pages 87-98 in ICRISAT (réd.), Proceedings of the Second International Workshop on Striga. 5-8 Oct. 1981, IDRC/ ICRISAT, Ouagadougou, Upper Volta. $\mathrm{Pa}$ tancheru, A.P., India.

Olivier, A., K.V. Ramaiah et G.D. Leroux. 1991. Selection of sorghum (Sorghum bicolor(L.) Moench) varieties resistant to the parasitic weed Striga hermonthica (Del.) Benth. Weed Res. 31: 219-225.

Ramaiah, K.V. 1983. Striga research at ICRISAT Upper Volta Center. Pages 53-60 in ICRISAT (réd.), Proceedings of the Second International Workshop on Striga, 5-8 Oct. 1981, IDRC/ICRISAT, Ouagadougou, Upper Volta. Patancheru, A.P., India.

Ramaiah, K.V. 1984. Patterns of Striga resistance in sorghum and millets with special emphasis on Africa. Pages 71-92 in E. Ayensu et al. (réd.), Striga biology and control. Paris, CRDI/ICSU Press.

Ramaiah, K.V. 1986. ICSV 1002 HV. A promising sorghum variety from ICRISAT/Upper Volta. Pages 83-87 in FAO (réd.), Proceedings of the OAU/FAO Workshop on Striga. 23-27 Sept. 1985, Yaoundé, Cameroun.

Ramaiah, K.V. 1987. Breeding cereal grains for resistance to witchweed. Pages 227-242 in L.J. Musselman (réd.), Parasitic weeds in Agriculture. I: Striga. CRC Press Inc., Boca Raton, Florida, U.S.A.

Ramaiah, K.V. et C. Parker. 1982. Striga and other weeds in sorghum. Pages 291-302 in ICRISAT (réd.), Sorghum in the eighties : Proceedings of the International Symposium on Sorghum. 2-7 November 1981, Patancheru, A.P., India.

Ramaiah, K.V., V.L. Chidley et L.R. House. 1990. Inheritance of Striga seed-germination stimulant in sorghum. Euphytica 45: 33-38.
Saueborn, J. 1991. The economic importance of the phytoparasites Orobranche and Striga. Pages 137-143 in J.K. Ransom et al. (réd.), Proceedings of the Fifth International Symposium of Parasitic Weeds. Nairobi, Kenya: CIMMYT.

Vasudeva Rao, M.J. 1985. Techniques for screening sorghums for resistance to Striga. Information Bulletin no. 20. Patancheru, A.P., India : ICRISAT. 18 pp.

Waller, R.A. et D.B. Duncan. 1972. Corrigenda. A Bayes rule for the symmetric multiple comparisons problem II. J. Am. Stat. Assoc. 67: 253-255.

Williams, C.N. 1959. Resistance of Sorghum to witchweed. Nature 84: 1511-1512.

Wilson-Jones, K. 1953. Further experiments on witchweed control. I. The effect of hormone weed-killer applications at different rates and times on irrigated dura. Empire J. Expl. Agric. 21: 331-339. 